\title{
ANALYSIS OF THE RELATIONSHIP SYSTEM AND THE SUCCESS OF NETWORK MANAGEMENT ON LONG-TERM DEVELOPMENT CONTINUE TO THE CULTURAL HERITAGE HOM, PHRAE PROVINCE, THAILAND
}

\author{
Nawatrot Intem \\ Ph.D. Candidate (Education), Faculty of Education, Chiang Mai University, Thailand \\ DOI: 10.46609/IJSSER.2021.v06i01.022 URL: https://doi.org/10.46609/IJSSER.2021.v06i01.022
}

\begin{abstract}
This article is part of doctoral research in education, major in social studies, Faculty of Education, Chiang Mai University, by research studies on "Knowledge management Mor Hom wisdom for the sustainable inheritance of Phrae Province", this research is a combination of qualitative research and quantitative research. This article is presented to present the model of the relationship system and the success of network management towards long-term development to Hom cultural heritage, Ban Thung Hong, Phrae Province, Thailand. The samples used for responding to inquiries are(1) representatives of Mor Hom fabric enterprises in Phrae province by selecting a specific method and analyzing quantitative data with the average and standard deviation, as well as in-depth interviews. (2) Mor Hom product processing group representatives conduct specific group discussions between researchers and Mor Hom product processing group representatives. The findings from the study showed that the shared vision of the Mor Hom wisdom enterprise network for sustainable succession includes the opportunity for individuals with knowledge and experience both in the community and outside the community, to participate in the creation of a vision together with members and to the general public, to create a formal vision that will reflect the emphasis on quality development, which can be implemented and reflected in sustainable cooperation.
\end{abstract}

Keywords: Relationship System, Network Management, Cultural Heritage, Hom Culture

\section{Introduction}

"Hom" is one of the best qualities Hom in Thailand, located in Ban $\mathrm{Na}$ Khuha Community, one of the upstream communities that keep Hom from growing and exploiting forests and producing wet Hom raw materials. This time the community-made Hom Ban Na Tong, Chohae District, and Hom $\mathrm{Na}$ Khuha Community is considered a network community of BEDO with the potential 


\section{International Journal of Social Science and Economic Research}

ISSN: $2455-8834$

Volume:06, Issue:01 "January 2021"

to be the "upstream" of the "Ton Hom" plantation. With the right environment Ton Hom has a good quality, blue extract or Indican by $\mathrm{Na}$ Khuha Community, Suan Khuean Sub-district, Muang District, Phrae Province. The community is located in the middle of the valley at an altitude of 625 meters from moderate sea level, which is suitable for growing Ton Hom, a large group of quality leaves providing high meat and indigo content. The production of wet Hom is an important raw material for dyeing fabrics from natural colors of excellent quality, providing a beautiful dark blue color with a simple and shiny finish, according to Mr. Sawang Sriteu, a member of the Hom community enterprises "Na Khuha Community", Suan Khuean Sub-district, Muang District, Phrae Province. According to local wisdom, Hom stems and leaves are soaked in water for about 72 hours and then mixed with red and lime to a thick eruption that resembles a dark mud yolk called "Wet Hom". Also, BEDO has encouraged Na Khuha Community to develop as a water producer in the production of fresh Hom and wet Hom meat, to be a raw material for dyeing Mor Hom fabric from natural colors to the community. The company has cooperated with Maejo-Phrae University in Chalermprakiat, developing "Hom Wet Na Khuha" products under the brand "Hom Bpiak Lung Sa Wang".

However, Ban Thung Hong, Thung Hong Sub-district, Muang District, Phrae Province, is a community in the middle of the water and downstream production of Hom dye fabric, "Mrs.Ngiam" or "Mrs. Prapapan Sritarai", which is a community enterprise Mor Hom, Thung Charoen, natural dyeing at Ban Thung Hong. It is said that BEDO has promoted the use of natural Hom meat from Ban Na Khuha which is a good quality wet Hom, used to dye shirts, tiedye fabrics, fabric bags, and souvenirs instead of chemical colors.It also opens a learning center that tells the story of "Mor Hom natural dyeing", from the natural dyeing process, the unique fabric design in various ways, to the use of the pattern on the fabric for tourists and interested parties to dye themselves.It has become a turning point and a new kind of selling point that generates revenue for community enterprises, averaging 40,000 baht per week or more than 100,000 baht per month.In product development and packaging, BEDO has partnered with Maejo-Phrae University to encourage the community to develop a more diverse product model, to expand the marketing channels.For example, Hom powder products developed to transform into powders and brand "Hom Pong Na Tong", and "Shirt Kit, and Handkerchief Kit Products", brand "mor hom pa ngiam" is convenient to use and easy to transport. Moreover, customers can dye their fabrics."Watercolor Coloring Kit Product" Brand "Rak Hom" to meet the demand for art lovers, focusing on art, which is a new target market group that is different from the one that uses Mor Hom to dye the fabric alone.

However, BEDO has the idea to honor Hom of Phrae province in Thailand, Thailand's best quality products are internationally recognized. As well as supporting and encouraging Hom growers, the meat processing Hom, inheriting local wisdom not to be lost, helping to preserve 


\section{International Journal of Social Science and Economic Research}

ISSN: $2455-8834$

Volume:06, Issue:01 "January 2021"

Hom genetics in the natural forest, as well as creating jobs and increasing income for sustainable communities.The production of Mor Hom fabric of Ban Thung Hong, Phrae Province. In addition to making a wide range of clothing and products, it also creates a career and generates income for the community, the community values nature and knows how to use it in everyday life properly. As well as maintaining new wisdom and knowledge development, the transmission from generation to generation in the community. As well as the community's management, such as grouping together to form a cooperative, etc.Creating the economy in a new context according to the strategy to generate income as a tool of conservation or, on the contrary, use conservation as a mechanism for generating three incomes, this includes creating forward-looking and sharing values. The governor said "Hom" is an attempt to get business escaping from nature as an example of bio-economy or community-level bio-economy.This consists of (1) the use of community-owned biodiversity and local wisdom as the main raw material (2) biodiversity and environmentally friendly productionand (3) to allocate some of the proceeds to conserve biodiversity.

Therefore, MorHom fabric or indigo dyed fabric is not just a plain dark blue fabric, but Mor Hom is also a fabric that is unique in its unique smell and color, unlike fabrics made from the factory, the only fabrics that are made of the fabric are manufactured, it also encompasses the dedication of both the strength and the heart of the weaver and the dye. As a result, Mor Hom fabrics, as well as a wide range of products from the Mor Hom fabric of Phrae province, which are dyed with natural indigo, have become one of the most popular products for both Thais and foreigners. In particular, European countries and Japan are not sufficiently manufactured to meet demand.This is because Mor Hom fabric or indigo dyed fabric is a natural product, which is a unique lyrical craft.

According to the research study titled "Mor Hom Knowledge Management for Sustainable Succession of Phrae Province", this article is presented to propose a model of the relationship system and the success of network management on the long-term development of Hom cultural heritage, Ban Thung Hong, Muang District, Phrae Province of Thailand.This is to know the shared vision of the Mor Hom wisdom enterprise network for sustainable succession, which provides opportunities for people with knowledge and experience both in the community and outside the community, to participate in the creation of a vision with members and to the general public, creating a formal vision that reflects the emphasis on quality development and practicality, as well as reflecting sustainable cooperation.

\section{Research Objectives}

To propose a model of the relationship system and the success of network management towards long-term development to Hom cultural heritage, Ban Thung Hong, Muang District, Phrae 
Province of Thailand.

\section{Conceptual Framework}

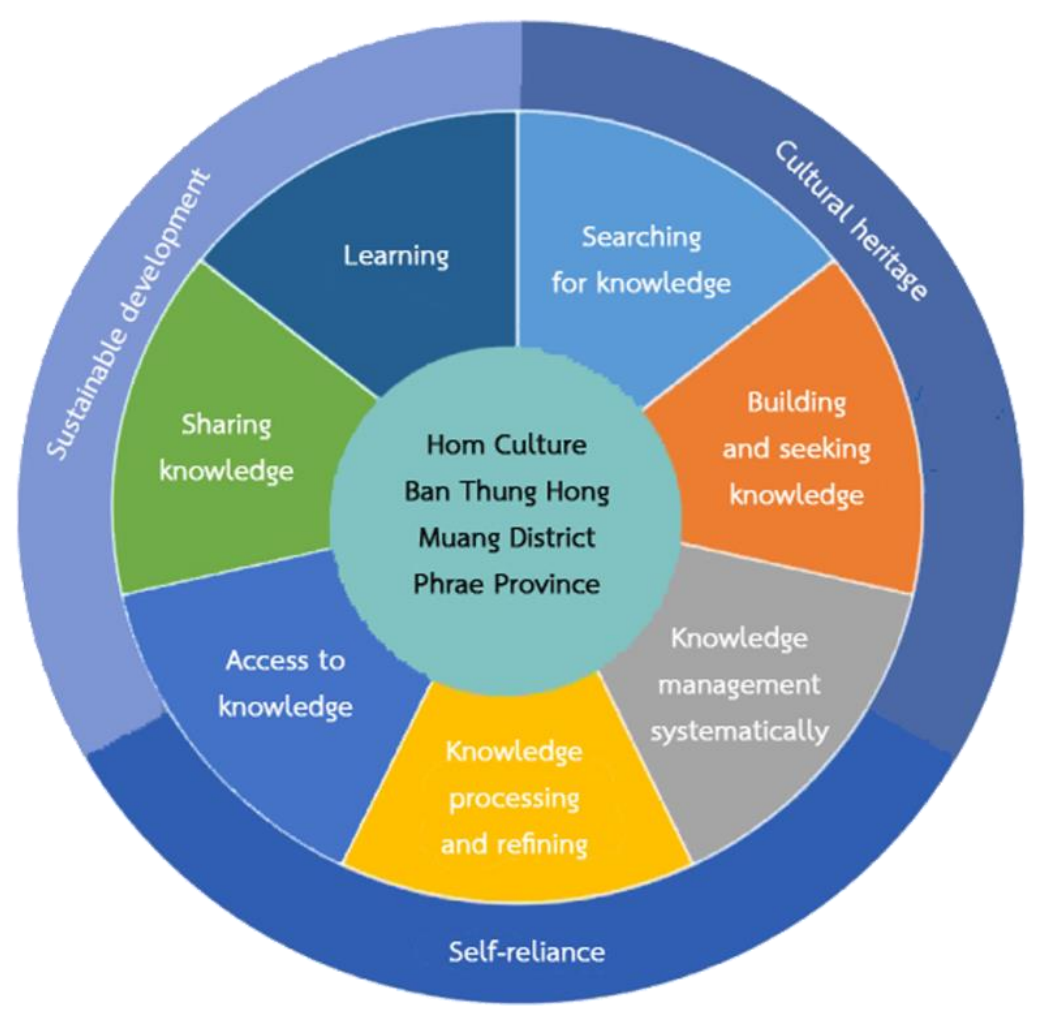

Figure 1: Conceptual framework of the research

\section{Literature Review}

\section{Concept of local wisdom}

"Folk Wisdom" is one of the most important social ideas; a large, modest, and long-lasting society must be together in all societies, Thai society is an old society, so there appears to be a lot of wisdom. These pearls of wisdom, in addition to being Thai, are one of the most important Thai indicators, and the development of the people of that country. This is because wisdom is a delicate thing, a nation that thrives on a peaceful, peaceful, and independent society so that they can be creative and have their unique wisdom. Mention of folk wisdom often refers to 


\section{International Journal of Social Science and Economic Research}

ISSN: $2455-8834$

Volume:06, Issue:01 "January 2021"

abstraction, the elite, and the end of the social institution. This is a component of each social culture, and social institutions must exist with a group called the social organization, where this social organization is independent, not requiring a cohesive social institution. This social and social organization, when sealed together, is a unified unit called the social structure. This can be separated into sections based on specific functions, each section has a specific function called a social unit, so each social unit consists of a person (multiple people) and a stereotype for their practice, to make it clearer, each social unit consists of: the four elements are: (1) social position (2) duties (3) behavioral patterns, this includes beliefs, knowledge, values, ideology, and social norms, and (4) objects from the structure of this social unit are part of the essence or many things.It may also be added that wisdom is a knowledge that is all connected, occupation, economy, well-being, spending, education, and culture. (Ruangdet Pankhiankhat, 1999)

\section{Context, the operating status of Mor Hom fabric community center, Phrae province in Thailand}

The Community Center Promotion Act B.E.2001 has established the department of agricultural promotion as the center for the promotion of community centers. The department of agriculture is responsible for certifying the status of the community and network centers and is the secretary's office; the community center promotion committee has three roles:(1) being a registered entity, revoking community centers and networks, and dissolution (2) as secretary's office community center promotion committee and (3) to promote community centers and networks in a unified manner. The guidelines for promoting community centers are as follows.

(1) The promotion of community centers and networks will be carried out in the manner of integration both within the agency and in cooperation with all parties, to jointly promote and encourage the community to be strong and self-reliant, the department of agriculture acts as the centerpiece to bring about the power to drive collaboration with parties.

(2) Application for registration of community centers and networks will be voluntary and community readiness, it is not targeted at the number of community centers that must be registered each year, but it is important to understand the public and the community about the benefits, how and procedure for registering a community center under the community center promotion Act B.E.2001 thoroughly.

(3) Officers in all public and private sectors involved should play a role in coordinating with community centers on welding, to achieve facilitator, synchronization to achieve catalyst and networker.

(4) Promoting community centers will focus on promoting the learning process and developing the potential of the community to manage community capital, both as money, resources, wisdom, 


\section{International Journal of Social Science and Economic Research}

ISSN: $2455-8834$

Volume:06, Issue:01 "January 2021"

and culture for self-reliance, and supporting community affairs rather than supporting factors and money to be free, community.

(5) Developing community center information system and network to establish information and knowledge links between community centers and networks, and to link information between community centers and related support agencies to develop underlying community centers in all provinces in 2005 .

\subsection{Community center grants}

Community centers have both funding and natural resources such as soil, water, forests, and productive grants, knowledge grants, wisdom, skills, cultures, social grants, or fraternity, community trust, community member relationship networks, and community centers to fund themselves, but they do not deny external funds based on new ideas: self-reliance, self-reliance, community funding, and community funding. While the community has resources, knowledge, intelligence, and others, which can be interpreted as money or invaluable, the need to increase capital to invest in community centers can be borrowed from the government savings bank, the bank for agriculture and agricultural cooperatives, which the bank for agriculture and agricultural cooperatives have budgeted up to 10,000 million baht, to support community centers or funds with policies to assist community centers.

\subsection{History of Mor Hom fabric}

"Mor Hom fabric" is an important local fabric of Phrae Province in Thailand. The word "Mor Hom" can be written in many ways. Whichever will be used but the true meaning is the same in every word. Indigo-colored clothing born from the wisdom of Thai ancestors is a cotton fabric that is woven and dyed with indigo from Ton Hom to produce the same color throughout the entire piece. Nowadays, it is cut into products such as trousers, skirts, shirts, and handkerchiefs. Festivals such as Loy Krathong, Songkran, New Year's Day, Annual Merit Festival, Phra That Festival, Tourism, and more, "Mor Hom" is popular for the wear of northern people and people all over the country, which represents a unique Thai fabric that is suitable for all genders. "Mor Hom" is a northern dialect. It's caused by the words "Mor" and "Hom", but many call it distorted. Which, it can be called, but the meaning is the same: the clothes were born of the wisdom of the ancestors in the form of indigo as a fallen plant, which Thung Hong residents, Thung Hong Sub-district, Mueang Phrae District, Phrae Province, Thailand. The stems and leaves are fermented in a pot that contains water in an ancient process and allows the indigo water to be dyed into indigo it's called "Mor Hom fabric". This is a cloth that people in Phrae and people all over the country use all genders, ages, not even foreigners. This can be seen that foreigners have very fond of Mor Hom fabric. 
International Journal of Social Science and Economic Research

ISSN: 2455-8834

Volume:06, Issue:01 "January 2021"

\subsection{Establishment of Mor Hom fabric community center of Ban Thung Hong}

This is due to the integration of people in the community to manage the community's funds creatively, by people in the community to respond to self-reliance and self-sufficiency of families and communities, due to community funding. This is due to the integration of people in the community to manage the community's funds creatively, by people in the community to respond to self-reliance and self-sufficiency of families and communities, due to community funding.This includes grants focused on natural resources, soil, water, yielding capital, knowledge grants, skills, traditions, cultures, people, fraternity, and community trust and grants.It was established as a group of Mor Hom Community Center, a natural dyeing field on August 5, 2005, and was registered on October 7, 2005, with 7 members starting to establish, Currently, there are 25 members, with a home office at 291 Moo 5, Thung Hong Sub-district, Muang Phrae District, Phrae Province. Most members work in making Mor Hom fabric produced from the factory. Nowadays, the wisdom of the ancestors of the Puan Thung Hong people Transmitted from generation to generation to make Mor Hom fabric natural color. It is a conservation of the wisdom of the villagers in the making of natural dye textile handicrafts. The development of Mor Hom fabric to the international community and to preserve the identity is sustainable and inherited.

Unique/Featured Products Mor Hom fabric Ban Thung Hong, Phrae Province, Mor Hom fabric is a natural cotton fiber dyed naturally. This is a local plant blended perfectly with the traditional dyeing method, through the process of hand-woven, mixed with local civilization and environmentally friendly, originally built as a garment product It's called "Mor Hom Shirt". It has been developed internationally and as a suited product, which can create a unique product that is famous for being recognized by Thai shoppers and foreigners who come to visit and shop.

\subsection{Cognitive value of Mor Hom fabric}

According to a study of the documents relating to dyeing, the ancestors of the past have a deep landscape of knowledge that has been passed down to interesting wisdom: the selection of dyes and fabrics by choosing Ton Hom to dye and is widely used in the past to the present day. It is a fabric that is more manufactured and used than other fabrics because it is used as a garment in everyday life. "Mor Hom fabric" will have an indigo smell, these fabrics are rarely damaged by insects because they do not like the smell of indigo, considering that ancestors have chosen natural dyeing materials with insect-resistant properties, they have a mysterious and non-toxic fragrance for people, but insects do not like this smell.

Ban Thung Hong, Phrae Province of Thailand, has been inherited from ancestors to children, which was originally produced for use in the family to the present day, which has been produced 


\section{International Journal of Social Science and Economic Research}

ISSN: $2455-8834$

Volume:06, Issue:01 "January 2021"

so much that it can be produced as a profession that helps to generate income for families and communities, it also creates economic stability for the local community by creating pride in the community. Nowadays, Mor Hom fabric has become a symbol and commodity in Phrae Province, which is well known to the public, bringing a reputation and pride in the uniqueness of the people in the community and creating a sustainable balance between people and society and nature. Because the production process uses natural processes with natural materials, it does not affect the environment and nature, resulting in a proper and sustainable balance between people and society. As well as forming a group of established members, professional groups and members of the group or community to participate in beneficial activities and create unity in the community itself, working together to develop potential as a key mechanism for working with the community. Mor Hom fabric direction in the future of the production of Phrae Province of Thailand, in addition to making a wide range of clothing and products, it also creates a career and generates income for the community, it is something that the community values and recognizes the benefits of everyday life properly. This includes maintaining new wisdom and development of knowledge; passing from generation to generation in the community, as well as the community's management, for example, the integration to form a cooperative, etc. So, Mor Hom fabric It's not just a plain dark blue cloth, it is also a fabric that is not unique in terms of its unique smell and color, which is different from the fabric made from the factory, it also encompasses the dedication of both the strength and the heart of the weaver and the dye. As a result, Mor Hom fabric, as well as a wide range of products dyed with natural indigo, has become one of the most popular products for both Thais and foreigners, in particular, European countries and Japan are not sufficiently manufactured to meet demand.This is because Mor Hom fabric is a natural product, it is a handicraft with a unique color that is unique, it is another art that is worth preserving, promoting, and conveying to the generations of children. However, those who are most knowledgeable in the Mor Hom fabric are most likely to be between 40 and 50 years old.It is worrying that if future generations do not learn the wisdom of weaving or dyeing fabrics and not seeing the importance of choosing Mor Hom fabric products. Also, if there is no local curriculum in the teaching system for children and young people in the community, you will not be able to provide the local curriculum. Soon, Mor Hom fabric dyeing naturally will be left to the story, sad memory of the forgotten local wisdom of Thai society.

In other words, Mor Hom fabric is a pearl of folk wisdom that is worthy of education, conservation, and inheritance as a treasure of Phrae Province and the entire Thai people, from the production of Mor Hom fabric to the family, society, and exchange until it comes to production for sale. The conservation of natural dye fabrics is an incentive, making it a profession that generates income and economy for local communities to manage in many ways, such as the establishment of a consortium/club, in a research study on Mor Hom fabric and raw material supply as well as investment and distribution, etc. Therefore, it should be preserved for future 


\section{International Journal of Social Science and Economic Research}

ISSN: $2455-8834$

Volume:06, Issue:01 "January 2021"

generations to inherit the traditional process processes, as well as to apply and develop different models according to market demand, without losing the original identity of the Mor Hom fabric. This will preserve, promote, convey and inherit this valuable local wisdom as a cultural heritage, this will create love, tenacity, and pride in the identity of the community itself.Also, it can be used as a profession to raise income for families, communities, and the nation, which is unique to the Thai nationality that is passed on to the fabric as a cultural heritage that people of all professions are very attentive to and promoted, such factors encouraged villagers to occupation the Mor Hom fabric.A continuous succession of wisdom is important, each craft takes time to produce complex, high-cost, high-cost Thais, so Thais should support and use Thai-made products in the form of Mor Hom fabric.

\subsection{Future Mor Hom fabric direction}

Phrae Province of Thailand is the largest source of ton Hom and produces dyeing fabrics in the country, with the right environment and wisdom inherited from generation to generation, "Hom" is a sub lusty plant that grows well in high areas in light-accessed natural forests and near water sources, as well as very humid and cold throughout the year, but nowadays, Hom is found to be less in nature. Meanwhile, demand for Mor Hom fabrics has increased and chemical dyes have been used to replace Hom fabrics. Therefore, Phrae Province of Thailand has campaigned for the use of production processes that do not affect the environment and biodiversity, and when the community is professional and income- and has a portion of the proceeds back to conserve and restore Hom plantations. The province's work with the community this time, the community is ready to expand by conveying knowledge and processes as an example by the Phrae Provincial economic development agency, let the agency focus and come together to push for a greater expansion of the concept today, to create an economy in a new strategic context, "a monetization is a tool of conservation, or vice versa, using conservation as a mechanism for making money in three ways: value creation and forward value, and sharing". The governor of Phrae Province said Hom was "an effort to get Hom businesses Phrae Province in Thailand's to turn to Hom from nature". As an example of bio-economy or community-level bio-economy, that is, (1) the use of community-owned biodiversity and local wisdom as the main raw material (2) biodiversityfriendly production and environment, and (3) partial revenue sharing to conserve biodiversity sources.

\section{Methodology}

\section{Method of study}

Cultural research in the local knowledge management of Ban Thung Hong, Phrae Province, is mixed research, documentary research, and qualitative research. That is a link of information, 


\section{International Journal of Social Science and Economic Research}

ISSN: $2455-8834$

Volume:06, Issue:01 "January 2021"

opinions, and knowledge management Hom local wisdom, Ban Thung Hong, Phrae province, there are two types of studies:

1) Documentary studies conduct studies and collect information from relevant documents and evidence, books, research reports, relationship documents that demonstrate concepts, principles, background, knowledge management Hom local wisdom, Ban Thung Hong, Phrae Province.

2) Qualitative Research in a field study conducted an in-depth interview to learn about the management of Mor Hom wisdom in ban Thung Hong, Phrae Province. In the area where the case is, and the focus group discussion to study the management of Hom knowledge local wisdom, Ban Thung Hong, Phrae Province, as follows:

(1) To study and select temples, communities, local administrative organizations, Ban Thung Hong, Phrae Province, purposive sampling based on the importance of the subject is an organization that plays a role and relationship with the management of local wisdom knowledge, Ban Thung Hong, Phrae Province, there is one community, Ban Thung Hong, Phrae Province, totaling 18 people, and a small group discussion of 12 people from local government organization leaders, villagers, and community leaders from a specific sample selection.

(2) Study and gather information from in-depth interviews and sub-group meetings with monks, local government leaders, philosophers, and related community leaders.

(3) Conduct a conceptual analysis study, principles local wisdom knowledge management Ban Thung Hong, Phrae Province. In the manner of in-depth analysis, it focuses on the participation processes of those involved in conducting research.

(4) To summarize and present the results of the study, both in the field and in the field. It is analyzed based on the important aspects of the community context and knowledge of Mor Hom, Mor Hom knowledge analysis, Ban Thung Hong, and conservation analysis in various dimensions. The study focuses on the results of the research to be distributed to monks, local government administrators, villagers, community leaders, and those involved have been informed and further developed.

(5) Summary of research study and suggestions.

\section{Research area}

Cultural research, local knowledge management, Ban Thung Hong, Phrae Province, interviewed the leaders of monks, local government leaders, sage villagers, and community leaders, and the Mor Hom fabric enterprise group, Phrae Province which plays a role in the development of Hom culture, the management of knowledge local wisdom, Ban Thung Hong, Phrae Province. 
International Journal of Social Science and Economic Research

ISSN: 2455-8834

Volume:06, Issue:01 "January 2021"

\subsection{Population and sample}

\section{A. Quantitative}

(1) Population is the population of Mor Hom fabric community enterprise registered population of 100 people in Muang Phrae district.

(2) Samples of this research were Ban Thung Hong, Thung Hong Sub-district, Mueang Phrae District, Phrae Province, totaling 100 persons by a specific random sampling method, to study knowledge management local wisdom at Ban Thung Hong, Phrae Province.

\section{B. Qualitative}

The in-depth interviews include community leaders/representatives of the Mor Hom fabric/local government group. 18 people in Phrae Province. The study involved knowledge and management of the Mor Hom fabric product network of Phrae province and the samples that carried out the focus group discussion.

Because the researchers established a case study area in Thung Hong Sub-district, Muang Phrae District, Phrae Province, which leads the community, therefore, the focus group discussion was conducted by conducting a specific group discussion between the researchers, four Mor Hom product processing representatives, representatives/local government organizations and leaders in Thung Hong Sub-district 4 persons, 2 monks, and 2 people who own a wholesale retailer, totaling 12 people, to study Hom culture local knowledge management, ban Thung Hong, Phrae Province.

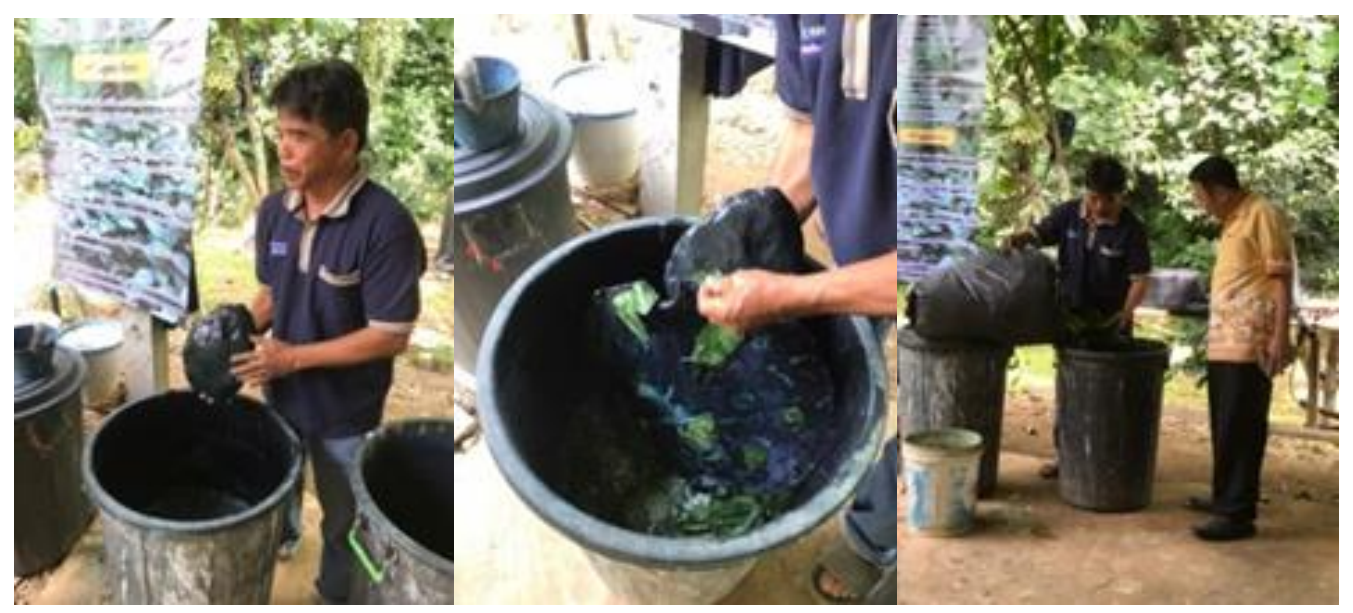

Figure 2: Visiting the area to observe the process of making raw broth 
International Journal of Social Science and Economic Research

ISSN: 2455-8834

Volume:06, Issue:01 "January 2021"

\section{Instruments used in research studies}

The tool used in this research is a questionnaire developed by the researchers following the framework of the local knowledge management culture Phrae Province. It aims to study context, community culture, and knowledge about Mor Hom and to study Mor Hom knowledge management. The data from the sample used in the research is divided into 3 episodes as follows:

Part 1: The respondent's general inquiries it is characterized by a closed-end consisting of gender, age, education level, occupation, and income.

Part 2: As a model for Hom culture local knowledge management, Ban Thung Hong, Phrae Province. The researchers divided the questions into two groups according to their objectives, with respondents having to comment on the questions given by the researchers.

\section{Information Collection}

(1) The researchers conducted a study of contextual documents, the current condition of Mor Hom fabric products, the concept of Hom culture knowledge management, local wisdom, Ban Thung Hong, Phrae province.

(2) Samples that responded to a questionnaire about Hom culture, management of local wisdom knowledge, Ban Thung Hong, Phrae Province, from representatives of Mor Hom fabric group, Thung Hong Sub-district, Muang Phrae District, Phrae Province, 100 people by a specific random method.

(3) The researchers conducted in-depth interviews for those involved, with important issues such as context, knowledge, and network management of Mor Hom fabric products, 18 people.

(4) Conduct focus group discussions by conducting specific group discussions between the researchers, representatives of the Mor Hom product processing group, 4 representatives/local government organizations, and 4 leaders in Thung Hong Sub-district, 2 monks, and the people who own the wholesale retailer 2 people, totaling 12 people, to learn about the relationship system and the success of Hom culture, the management of local wisdom knowledge, Ban Thung Hong, Phrae Province.

(5) Perform field notes and record audio using the virtual audio recording to record information obtained from in-depth interviews, specific group conversations. 


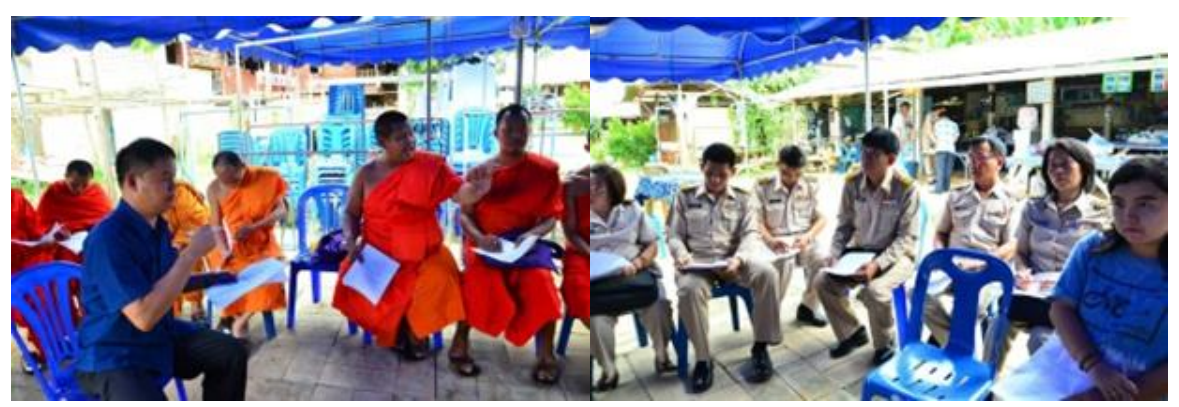

Figure 3: Small group discussion and study of fabric dyeing at Hom Ban Thung Hong

\section{Analysis of research data and statistics}

\subsection{Quantitative data analysis}

(1) The level of opinion on Hom culture, the management of knowledge, wisdom, local wisdom, Ban Thung Hong, analysis by looking at the mean and standard deviation of the score, from the distribution of community questionnaires in Thung Hong Sub-district, Phrae Province.

(2) Interpret mean based on Suwaree Siriphokaphirom criterion (2003).

(3) Information from interviews the researcher will data grouping according to the essence of the interview issue, then will content analysis technique.

\subsection{Qualitative data analysis}

(1) The researchers conducted in-depth interviews for those involved with important issues such as context, knowledge, Hom cultural management, knowledge management, local wisdom, Ban Thung Hong, Phrae Province.

(2) Summary of specific group discussions between researchers, representatives of the Mor Hom product processing group for those involved, to learn about the relationship system and the success of Hom culture, the management of local wisdom knowledge, Ban Thung Hong, Phrae Province.

\subsection{Statistics used in data analysis}

The confidence of the entire questionnaire was 0.80 using the Alpha Coefficient coefficient using Cronbach using the $\mathrm{Si} 2$ computer program, as well as the basic percentage and standard deviation. 


\section{International Journal of Social Science and Economic Research}

ISSN: $2455-8834$

Volume:06, Issue:01 "January 2021"

\section{Results and Discussion}

The results of the general analysis of the respondents $(\mathrm{N}=100)$ showed that the majority of respondents were female, accounting for $79.00 \%, 21.00 \%$ of respondents were aged 51 and over, the second was $40.00 \%$ between 41 and 50 years old, 36.00 percent were aged between 31 and 40 years old, $4.00 \%$ respectively, the majority of respondents had primary education, equivalent to $55.00 \%$. The second is a junior high school or equivalent $26.00 \%$, bachelor's degree $13.00 \%$, high school or equivalent and diploma $3.00 \%$ respectively. In this regard, the majority of respondents were in agriculture, accounting for $78.00 \%$, the second is to own business, $16.00 \%$ and $6.00 \%$ in the public service, respectively, and the average respondent earned 5,001-10,000 baht per month, equivalent to $76.00 \%$, the second line is $10,001-15,000$ Baht or $14.00 \%$ revenue of Baht 15,001 or above, equivalent to $10.00 \%$ respectively.

Hom cultural knowledge and local wisdom management at Ban Thung Hong, Phrae Province of Thailand, to collect data in both quantitative and qualitative, it can be analyzed for the relationship system and the success of network management on the long-term development of Hom cultural heritage, Ban Thung Hong, Muang District, Phrae Province of Thailand. As follows:

1) Common perspective recognition is the focus on natural Mor Hom colors, to maintain identity rather than the use of dyes from chemicals, coordination, and more serious cooperation. This opened up opportunities to study various places and exchange knowledge together (KM), as well as the creation of information systems from the level of the joint policy plan and the use of technology to facilitate and distribute it to the public, also need to adjust our attitudes and perspectives internationally.

2) The shared vision of the Mor Hom wisdom enterprise network for sustainable succession is to provide opportunities for people with knowledge and experience both in the community and outside the community, and to participate in creating a vision with members and disseminating them to the general public, as well as creating a formal vision that will reflect the emphasis on quality development, practicality, and sustainable cooperation.

3) The relationship and success of the Mor Hom wisdom enterprise network for sustainable succession, namely, to cooperate in proactive marketing and product expansion from different areas of the province and abroad, as well as to have ethical integrity to customers, to build confidence in the business, have a management system that generates revenue benefits for members and distributes revenue appropriately and fairly, as well as providing good service to customers to impress. 


\section{International Journal of Social Science and Economic Research}

ISSN: $2455-8834$

Volume:06, Issue:01 "January 2021"

4) The relationship and achievement system of member engagement network management, which has a wide range of networks such as the Mor Hom wisdom enterprise network for sustainable succession, namely unity, non-commercial quality, product quality, size, and price must be in the same direction, communication formally and informally, to have professional consultants to bring together all parties to develop, as well as to contribute to the real knowledge, skills and experience of operation and focus on members of the community and then expand to the outside of the community.

5) Relationship system and the success of network management, mutual strengthening of the Mor Hom wisdom enterprise network for sustainable succession. This is to work together cooperatively with an acceptable standard of performance assessment, seeking a variety of knowledge, exchanging, learning, and encouraging members and other activities, to bring together love and harmony.

6) Relationship system and the success of network management, the mutual dependency of the Mor Hom wisdom enterprise network for sustainable succession, in other seeing a large family business seeing customer service more critical to product quality to a higher standard than business coordination in a fast and efficient meeting place or doing business.

7) The relationship and success of the management of the Mor Hom wisdom enterprise network exchange network for sustainable succession, which includes interaction in lifestyle activities, cultural customs and traditions, horizontal interaction rather than vertical interaction, and more positive-negative interactions, as well as more concrete interactions than abstraction, as well as practical interactions rather than policies.

Therefore, according to a research study on the current context of Hom culture, the management of local wisdom knowledge, Ban Thung Hong, Phrae Province, Thailand, in the analysis of the relationship system and the success of network management towards long-term development, in conclusion, it's a conceptual plan, as follows figure 4: 
International Journal of Social Science and Economic Research

ISSN: 2455-8834

Volume:06, Issue:01 "January 2021"

\section{Culture knowledge management, local wisdom of Ban Thung Hong, Phrae Province of Thailand}
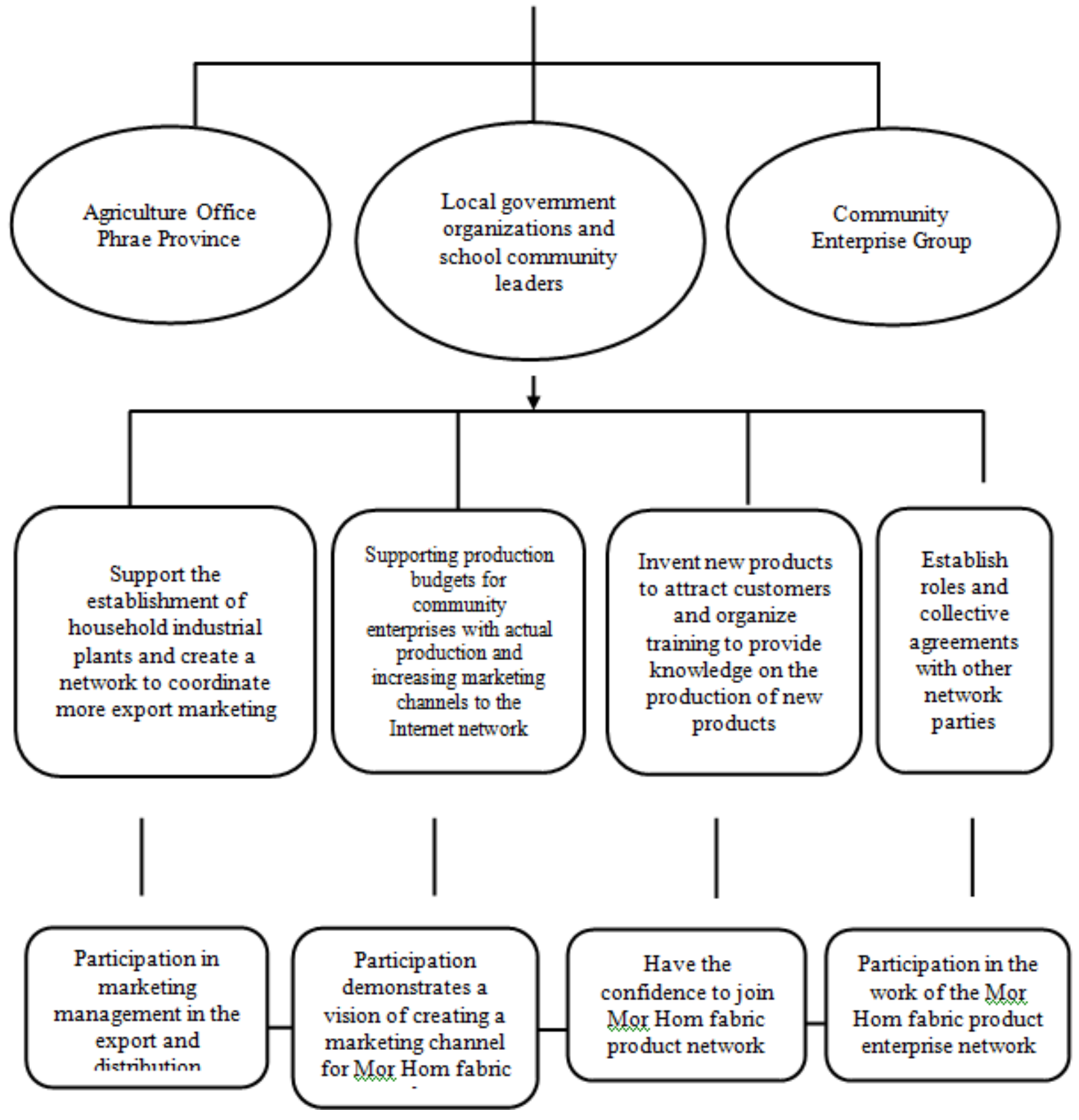
International Journal of Social Science and Economic Research

ISSN: 2455-8834

Volume:06, Issue:01 "January 2021"

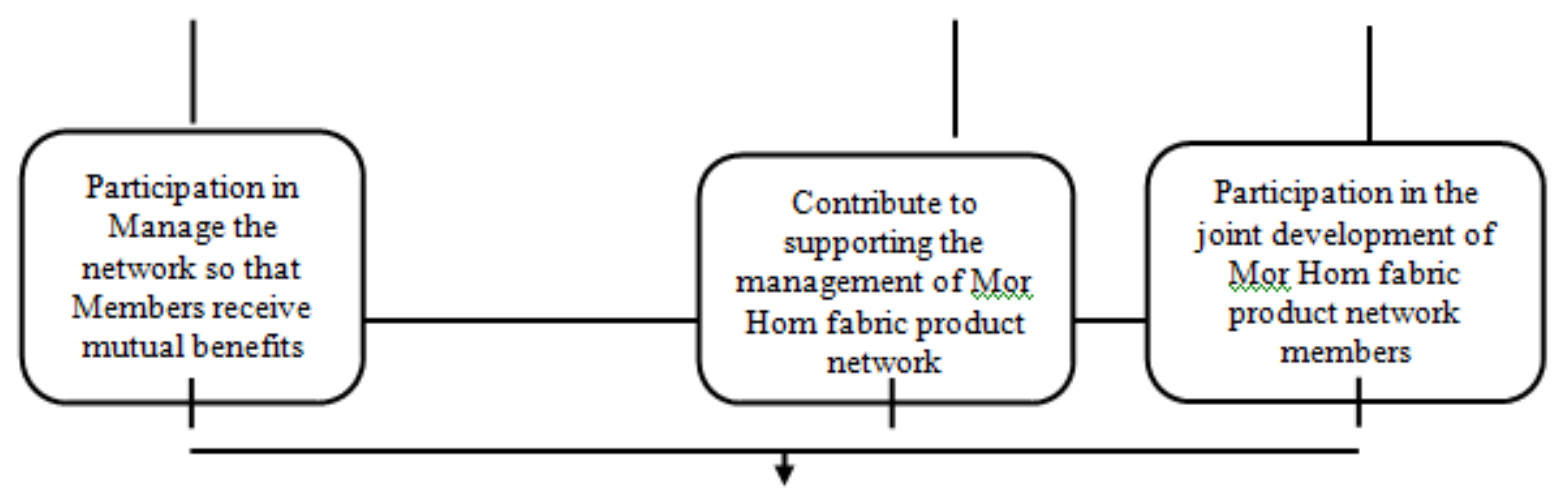

Offering knowledge and management system, Hom cultural network, knowledge management local wisdom Ban Thung Hong, Phrae Province of Thailand

\section{Discussion of results}

Based on the analysis of the relationship system and the success of network management on the long-term development of Hom cultural heritage, Ban Thung Hong, Muang District, Phrae Province of Thailand is as follows:

1. Relationship system and the success of network management, the perception side, mutual perspective of knowledge management, local wisdom, community enterprise network Mor Hom fabric, found that:

1) Community enterprises must focus on Mor Hom colors derived from natural Ton Hom to preserve identity rather than the use of dyes from chemicals.

2) Community enterprises need to be more coordinated and cooperative.

3) Community enterprises have the opportunity to study the best practice and exchange that knowledge together $(\mathrm{KM})$.

4) Community enterprises have established information systems from the level of the joint policy plan and should be used to facilitate and distribute them to the public.

5) Community enterprises need to adjust attitudes and perspectives that are universal. 


\section{International Journal of Social Science and Economic Research}

ISSN: $2455-8834$

Volume:06, Issue:01 "January 2021"

2. Relationship system and the success of network management aspects of the common vision of knowledge management, local wisdom, community enterprise network Mor Hom fabric, found that:

1) Provide opportunities for people with knowledge and experience both in the community and outside the community to participate in the creation of a vision with members of the Mor Hom fabric enterprises.

2) Vision should be distributed to the general public such as television, radio, newspaper, and internet.

3) It is best to create a formal vision, such as "Mor Hom 5 Stars, beyond the world".

4) Should create a vision that will reflect the emphasis on quality development.

5) A vision that can be implemented and reflected in collaboration.

3. Relationship system and the success of network management, the side of the benefits and mutual interests of knowledge management, local wisdom, community enterprise network Mor Hom fabric, found that:

1) Mor Hom fabric enterprises need to be proactively marketed, but cooperation is important.

2) Mor Hom fabric enterprises must expand from provincial and international areas.

3) Mor Hom fabric enterprises must have ethical integrity to customers to build confidence in the business.

4) Mor Hom fabric enterprises must have a benefits management system that generates income for members and distributes income appropriately and fairly.

5) Mor Hom fabric enterprises should provide good service to customers to impress, especially tourists.

4. Relationship system and the success of network management aspects, the participation of members, the widespread network of knowledge management, local wisdom, and community enterprise network Mor Hom fabric, found that:

1) Mor Hom fabric enterprises are not united, foreign traders; product quality, size, and price are not in the same direction.

2) Mor Hom fabric enterprises lack communication with each other formally and informally. 


\section{International Journal of Social Science and Economic Research}

ISSN: $2455-8834$

Volume:06, Issue:01 "January 2021"

3) Mor Hom fabric enterprises should have professional consultants to bring together all parties to develop for real participation.

4) Mor Hom fabric enterprises lack knowledge, skills, and operational experience.

5) Mor Hom fabric enterprises should focus on community members as a priority and expand to the outside of the community.

5. Relationship system and the success of network management, mutual strengthening of knowledge management, local wisdom, and community enterprise network Mor Hom fabric, found that:

1) Mor Hom fabric enterprises should be cooperative in a cooperative manner.

2) Mor Hom fabric enterprises should be evaluated following acceptable standards.

3) Mor Hom fabric enterprises should seek a wide range of knowledge.

4) Mor Hom fabric enterprises should exchange, learn and build morale to members.

5) Mor Hom fabric enterprises should have other activities to co-ordinate to achieve love and harmony.

6. Relationship system and the success of network management of mutual dependency of knowledge management, local wisdom, and community enterprise network Mor Hom fabric, found that:

1) Mor Hom fabric enterprises must be seen as a big family business.

2) Mor Hom fabric enterprises need to see how customer service is important.

3) Mor Hom fabric enterprises need to look at quality to meet the standards rather than a business.

4) Mor Hom fabric enterprises need to be coordinated in a fast and efficient manner.

5) Mor Hom fabric enterprises must have a meeting place or continue to do so.

7. Relationship system and the success of network management, exchange interaction of knowledge management, local wisdom, and community enterprise network Mor Hom fabric, found that: 


\section{International Journal of Social Science and Economic Research}

ISSN: $2455-8834$

Volume:06, Issue:01 "January 2021"

1) Mor Hom fabric enterprises must interact in lifestyle activities, customs, and cultural traditions.

2) Mor Hom fabric enterprises require more horizontal interaction than verticals.

3) Mor Hom fabric enterprises require more positive than negative interactions.

4) Mor Hom fabric enterprises require more concrete interaction than an abstraction.

5) Mor Hom fabric enterprises require more practical interaction than policy.

\section{Conclusion}

Hom cultural knowledge and local wisdom Management at Ban Thung Hong, Phrae Province of Thailand, the system of relationship and the success of network management is the long-term development of Hom cultural heritage, Ban Thung Hong, Muang District, Phrae Province of Thailand, as follows:

1. In terms of context and cultural problems, Hom in the field of knowledge management, local wisdom, it is found that the place should be provided in the parking lot, to facilitate customers and to beautifully adjust the landscape of the shop area. The raw material should be used as a natural dye adequately and should be priced fairly and appropriately, and the same standard should be set and the quality of the paint and sewing standards should be maintained, also focus on good service to impress customers and to include concrete enterprise members.It should be regularly promoted and accessed by all media, and the database system should be systematically and up-to-date, and should be studied in other agencies or enterprises with good performance, community learning centers should be managed to demonstrate or be a source of learning to the general public and should be supported by government agencies such as local government organizations that are diverse to drive the implementation of the enterprises.

2. Hom cultural network management in the field of knowledge management, local wisdom, it found that the common perception of the view was to focus on natural Mor Hom colors, to maintain identity rather than the use of dyes from chemicals, and in terms of coordination and cooperation more seriously, by opening up opportunities to study various locations and exchange knowledge together (KM), as well as the preparation of information systems from the level of the policy plan together, the use of technology to facilitate and distribute to the public and the shared vision of Mor Hom fabric enterprise network management is to provide opportunities for people with knowledge and experience both in the community and outside the community, and to participate in the creation of a vision together with members and to the general public, as well as 


\section{International Journal of Social Science and Economic Research}

ISSN: $2455-8834$

Volume:06, Issue:01 "January 2021"

to create a formal vision that will reflect the emphasis on quality development, practicality, and sustainable integration.

3. The relationship system and achievements of Hom culture management in the field of knowledge management, local wisdom, benefits network, and the common interests of Mor Hom fabric enterprise network management, namely proactive marketing cooperation, as well as the expansion of goods from provincial and international areas, and moral ethics to customers to build confidence in the business, as well as a benefits management system that generates revenue and distributes revenue appropriately and fairly, and provides good service to customers to impress.

4. Aspects of the relationship system and the success of Hom cultural management in the field of knowledge management, local wisdom. There is a wide network of participation of members of the Mor Hom fabric enterprise network management, namely the unity of non-foreign traders, product quality, size, and price must be in the same direction, communicating formally and informally, as well as having professional advisors to bring together all parties to develop the potential for real participation, for real engagement, operational skills, and community focus, and then expand to the community outside.

5. The system of relationship and achievement of Hom cultural management in the management of knowledge, local wisdom, the mutually strengthening network of Mor Hom fabric enterprise network management, namely, cooperative collaboration, evaluation of performance according to acceptable standards, and the pursuit of diverse knowledge, exchange, learn and morale, and other activities to co-ordinate to achieve love and harmony.

6. Aspects of the relationship system and the success of Hom culture management in the management of knowledge, local wisdom, Mor Hom fabric enterprise network management is seen as a large family business, seeing customer service as critical to product quality to meet the standards of business, coordination in a fast and efficient manner, and there's a meeting place or a continuation of the meeting.

7. Aspects of the relationship system and the success of Hom culture management in the management of knowledge, local wisdom, exchange interaction network of Mor Hom fabric enterprise network management, and interaction in lifestyle activities, traditions, and cultural traditions, horizontal interaction over the vertical, more than negative interactions, including concrete, more abstract and more practical interactions than policies.

\section{Suggestion}




\section{International Journal of Social Science and Economic Research}

ISSN: $2455-8834$

Volume:06, Issue:01 "January 2021"

The findings are based on research. Policy recommendations on government agencies such as local government organizations, provincial cultures, universities, and public and private sectors, can be used to provide knowledge from this research as a framework for policy designation. In the development of the Mor Hom fabric of Phrae province in Thailand to be sustainable.

Including practical feedback, practical action is the second priority of policy operations, because effective and effective success is not the only policy action. It also requires practical action to solve the problem effectively Mor Hom's knowledge of wisdom should be developed to the operator of Mor Hom fabric to be open to the situation. In a development that can strengthen the Mor Hom fabric community, it can be sustainable to be self-reliant. This should accelerate the promotion of both the public, private sector, and the cooperation network between the agencies to participate in information and strengthen knowledge management for the succession of Mor Hom, Ban Thung Hong community, Phrae Province in Thailand to be sustainable.

\section{References}

Drucker \& Perter, F. 1998. The coming of the new organization. In Harvard Business Review on knowledge management, 1-20. Boston, MA: Harvard Business School Publishing.

Kanjanarangsinont, K. 2011. “Sufficiency Economy Benefits Research Project”. Office research Research Fund (TRF).

Kalseth, K. 1997. Knowledge management: Putting information and people'scompetence to work together. FID News Bulleting 47, 7/8 (July August): 191.

Panchiankhat, R. 1999. A Study of Place Names in Thai and Chan Languages in Thailand ". In the journal language and culture Year 14 No. 2 July - December.

Pongpit, S. 2001. Community Enterprise Handbook. Bangkok: Power of wisdom.

Pongpit, S. 2002. Network. Bangkok: Power of wisdom.

Pongpit, S. 2002. Thinking base from the master plan to community enterprises. Bangkok: Charoenwit Printing.

Phra Maha Suthit Apak Ro. 2001. Natural Network, Knowledge and Management. Bangkok: Lak Publishing Publishing House.

Puangngam, K. 2007. A new dimension of local governance, the vision of decentralization and local administration.Bangkok: Saematham. 
International Journal of Social Science and Economic Research

ISSN: 2455-8834

Volume:06, Issue:01 "January 2021"

Ruggles \& Rudy, L. 1997. Tools for knowledge management: An introduction. In Knowledge management tools, 1-8. Boston: Butterworth-Heinemann.

Ramos, L.A.A \& Fletcher, L.B. 2017.Planning for Rural Development: Rural Development with Popular Participation.Lowa: Department of Economics, Lowa State University.

Siriphokaphirom, S. 2003. Educational research. Lopburi: Thepsatri Rajabhat Institute.

Taylor, F.W. 1998. The principle of scientific management. New York: Harper Bros.

Wiratnipawan, W. 2001. Administration and administration of constitutional organization development and Government agencies.Bangkok: Dharmniti Co., Ltd. 